\title{
Function of a novel nasal protrusion for oral-shelling within an adaptive radiation of pupfishes
}

5

6

7

8

9

10

11

12

13

14

15

16

17

18

19

20

21

22

23

24

25

26 Short title: Function of nasal protrusion

27 keywords: adaptive radiation, speciation, novelty, performance, durophagy, craniofacial,

28 foraging

29 Abstract: 293 words, Main text plus references: 4988 words, 3 Figures, Supplemental Figures

30 S1\&S2, and 1 Supplemental video S1

31
'Department of Integrative Biology and Museum of Vertebrate Zoology, University of California, Berkeley, CA 94720, USA

2Department of Biology, University of North Carolina at Chapel Hill, 120 South Rd, NC 27599, USA

Corresponding Author: chmartin@berkeley.edu 


\section{Significance Statement}

33 Specialization on hard-shell prey items (i.e. durophagy) is a common dietary niche among fishes.

34 Oral shelling is a rare technique used by some durophagous fish to consume prey items like

35 snails; however, adaptations for oral shelling are still unknown. Here, we document the first

36 evidence of oral shelling in a cyprinodontiform fish, the durophagous pupfish (Cyprinodon

37 brontotheroides), and experimentally test whether its novel nasal protrusion is an adaptation for

38 oral shelling using hybrid feeding trials.

39

40

41

42

43

44

45

46

47

48

49

50

51

52

53

54 


\section{Abstract}

56 Dietary specialization on hard prey items, such as mollusks and crustaceans, is commonly

57 observed in a diverse array of fish species. Many fish consume these types of prey by crushing

58 the shell to consume the soft tissue within, but a few fishes extricate the soft tissue without

59 breaking the shell using a method known as oral shelling. Oral shelling involves pulling a

60 mollusk from its shell and may be a way to subvert an otherwise insurmountable shell defense.

61 However, the biomechanical requirements and potential adaptations for oral shelling are

62 unknown. Here, we test the hypothesis that a novel nasal protrusion is an adaptation for oral

63 shelling in a durophagous pupfish (Cyprinodon brontotheroides). We first demonstrate oral

64 shelling in this species and then predicted that a larger nasal protrusion would allow pupfish to

65 consume larger snails. Durophagous pupfish are found within an endemic radiation of pupfish on

66 San Salvador Island, Bahamas. We took advantage of closely related sympatric species and

67 outgroups to test: 1) whether durophagous pupfish shell and consume more snails than other

68 species, 2) if F1 and F2 durophagous hybrids consume similar amounts of snails as purebred

69 durophagous pupfish, and 3) to determine if nasal protrusion size in parental and hybrid

70 populations increases the maximum diameter snail consumed. We found that durophagous

71 pupfish and their hybrids consumed the most snails, but did not find a strong association between

72 nasal protrusion size and maximum snail size consumed within the parental or F2 hybrid

73 population, suggesting that the size of their novel nasal protrusion does not provide a major

74 benefit in oral shelling. Instead, we suggest that nasal protrusion may increase feeding efficiency,

75 act as a sensory organ, or is a sexually selected trait, and that a strong feeding preference may be

76 most important for oral shelling. 


\section{Introduction}

79 Dietary specialization is thought to be one way to reduce competition for a food source or to

80 forage more optimally (Pyke 1984; Futuyman and Moreno 1988; Robinson and Wilson 1998).

81 One form of dietary specialization, especially among fishes, is the increased consumption of

82 hard-shelled prey items, such as mollusks and crustaceans (hereafter referred to as durophagy),

83 and both freshwater and marine fishes include durophagous specialists. There are two main ways

84 that fish consume hard-shelled prey items: First, fish may crush or break the outer shell to

85 consume the soft tissue within. Some fishes, such as black carp (Mylopharyngodon picesus),

86 pumpkinseed sunfish (Lepomis gibbosus), redear sunfish (Lepomis microlophus), black drum

87 (Pogonias cromis), Florida pompano (trachinotus carolinus), and the black margate

88 (Anisotremus surinamensis), use their pharyngeal jaws to crush the shells of snails and other

89 mollusks in order to consume them (Lauder 1983; Grubich 2003; Gidmark et al. 2015). Others,

90 such as the striped burrfish (Chilomycterus schoepfi), use their fused oral teeth to manipulate and

91 crush shells (Winterbottom 1974; Ralston and Wainwright 1997). The biomechanical constraints

92 of crushing hard shells is well documented in fish. For example, body mass $(\mathrm{g})$, bite force $(\mathrm{N})$,

93 and pharyngeal jaw gape size are understood to limit the upper size of prey in the Caribbean

94 hogfish (Lachnolaimus maximus), where larger fish generally produce both larger gapes and

95 increased crushing force, allowing them to crush larger or thicker shells (Wainwright 1987,

96 1991). Similarly, the upper prey size consumed by black carp is limited by 1) the amount of

97 force produced by its pharyngeal jaw closing muscle (medial levator arcus branchialis $V$ )

98 (Gidmark et al. 2013) and 2) the size of the pharyngeal jaw gape (Gidmark et al. 2015).

99

An alternative and much rarer method of consuming hard-shelled prey, primarily

100 documented in cichlids endemic to Lake Malawi (Metriaclima lanisticola), Lake Victoria 
101 (Hapochromis. xenognathus, H. sauvagei and Macropleurodus bicolor), and Lake Edward (H.

102 concilians sp. nov., H. erutus sp. nov. and H. planus sp. nov), is to extract the soft tissue of the

103 gastropod from its shell via wrenching or shaking, known as 'oral shelling' (Slootweg 1987;

104 Madsen et al. 2010; Lundeba et al. 2011; Vranken et al. 2019). It is typically thought that oral

105 shelling is a way to circumvent the force and pharyngeal gape size requirements for consuming

106 large mollusks because oral shelling does not require a fish to break a mollusk's shell; however,

107 very few studies have investigated oral shelling in general (but see: Slootweg 1987; De Visser

108 and Barel 1996) nor have they investigated adaptations for oral shelling.

109 One possibility may be that fish use morphological adaptations to create a mechanical

110 advantage during oral shelling. For example, one hypothesis is that the fleshy snout of

111 Labeotropheus cichlids is used as a fulcrum, allowing fish to more easily crop algae from rocks

112 versus the bite-and-twist method observed in other cichlid species (Konings 2007; Conith et al.

113 2018), and specifically that increased snout depth may help create this mechanical advantage

114 (Conith et al. 2019). A similar method may be used during oral shelling to amplify force while

115 removing snails from their shells. Thus, we predicted that larger nasal fulcrums should provide

116 greater mechanical advantage for successfully oral shelling larger prey.

117 The durophagous pupfish (Cyprinodon brontotheroides) is an excellent species for testing

118 whether a novel morphological trait provides a mechanical advantage for oral shelling.

119 Durophagous pupfish are found within an adaptive radiation of pupfish endemic to the

120 hypersaline lakes of San Salvador Island, Bahamas, which also includes a generalist pupfish $(C$.

121 variegatus) and a scale-eating pupfish (C. desquamator; Martin and Wainwright 2011, 2013a).

122 Geological evidence suggests that the hypersaline lakes of San Salvador Island, and thus the

123 radiation itself, are less than 10,000 years old (Hagey and Mylroie 1995; Martin and Wainwright 
$1242013 b, 2013 a$ ). Phylogenetic evidence also indicates that: 1) generalist pupfish found outside San

125 Salvador Island are outgroups to the entire San Salvador clade, and 2) that durophagous pupfish

126 cluster near generalists from the same lake populations, indicating that there is extensive

127 admixture between these young species (Martin and Feinstein 2014; Martin 2016; Lencer et al.

128 2017; Richards and Martin 2017). Gut content analyses indicated that durophagous pupfish

129 consume approximately 5.5 times the number of mollusks and crustaceans (specifically

130 ostracods) as generalists and fewer shells, suggesting that durophagous pupfish may be orally

131 shelling their prey (Martin and Wainwright 2013b). In addition to their dietary specialization,

132 durophagous pupfish also possess a novel nasal protrusion not observed in other pupfish species

133 (Martin and Wainwright 2013a). This nasal protrusion is an expansion of the maxilla, and

134 extends rostrally over the upper jaws (Hernandez et al. 2018). It is plausible that this nasal

135 protrusion is an adaptation for oral shelling used by the durophage as a fulcrum.

136 We investigated oral-shelling behavior in the laboratory and tested if the nasal protrusion

137 of durophagous pupfish is an adaptation for oral shelling. We measured snail consumption across

1386 groups in the laboratory: outgroup generalists, generalists from San Salvador Island, scale-

139 eaters, durophages, and F1 and F2 durophage hybrids (produced by crossing purebred

140 durophages and generalists in the lab). If the novel nasal protrusion is adapted for oral shelling,

141 we expected that durophages would consume significantly more snails than generalists and scale-

142 eaters. We also expected that F1 hybrids would show intermediate snail consumption between

143 the parental species and that F2 hybrids would show greater variation in snail consumption

144 compared to parental species. To directly tie nasal protrusion size to snail-shelling performance,

145 we also investigated whether individuals with larger noses could consume larger snails in lab-

146 reared populations of both durophages and F2 hybrids. Ultimately, we found that, contrary to our 
147 predictions, purebred durophages, F1, and F2 hybrids all shelled significantly more snails than

148 other pupfish species and we did not find evidence that larger nasal protrusion allowed

149 durophages to consume larger snails. Instead, we discuss alternative explanations for the novel

150 nasal protrusion such a putative function in foraging efficiency, sexual selection, olfaction, or

151 increased area for superficial neuromasts.

153 Methods

154 Collection and Care

155 During the summer of 2017, we used seine nets to collect generalist, durophage, and scale-eater 156 pupfishes from Crescent Pond, Little Lake, Osprey Lake, and Oyster Pond (San Salvador Island, 157 Bahamas). We transported fish back to the University of North Carolina, Chapel Hill, where they 158 were maintained in mixed-sex stock tanks (37-75 1) in approximately $26^{\circ} \mathrm{C}$ water at 159 approximately 5-10 ppt salinity (Instant Ocean salt mix). In the lab, we produced F1 and F2 160 hybrid offspring using snail-eater and generalist parents. Wild caught individuals were also 161 allowed to breed and produced F1-F3 purebred offspring. Hybrid and purebred offspring were

162 used in our feeding assays. We fed all fish a diet of commercial pellet foods, frozen bloodworms, 163 and mysis shrimp daily.

164 We also maintained a colony of freshwater sinistral snails (Physella sp.). We kept snails

165 in a 7 liter stock tank containing the same water used in pupfish tanks. All snails were acclimated 166 to $5-10 \mathrm{ppt}$ salinity for at least 48 hours before being used in a feeding trial. We fed snails a diet

167 of bloodworms every 48 hours. We ran multiple control trials without fish alongside feeding 168 trials to track natural snail mortality rates. 


\section{Morphological Measurements}

171 We measured standard length of each fish by measuring the distance from the tip of the upper

172 jaw to the posterior end of the hypural plate. We also measured nasal protrusion size for a subset

173 of fish (9 generalists, 50 durophages, 17 F1 hybrids, and 62 F2 hybrids) using image processing

174 software (Schindelin et al. 2012). Scale-eating pupfish do not exhibit even marginal nasal

175 protrusion, and therefore we did not include them in this analysis. We measured fish nasal

176 protrusion size by drawing a tangent line aligning the most anterior dorsal point of the premaxilla

177 with the neurocranium and measuring a perpendicular line at the deepest part of the nasal region

178 (Figure 1C).

179

180 Feeding Assay

181 We quantified the number of snails consumed by all three species of pupfish and hybrids using

182 feeding assays. Prior to a feeding assay, fish were removed from stock tanks and isolated in $2 \mathrm{~L}$

183 trial tanks which contained one synthetic yarn mop to provide cover for the fish. We allowed fish

184 to acclimate in trial tanks for at least 12 hours before the start of a feeding assay. After the

185 acclimation time, we haphazardly chose 5 snails from our snail stock tank and added them to

186 each feeding assay tank. We added one bloodworm to each tank to ensure that even fish which

187 did not consume any snails had an adequate diet. Fish were allowed to feed freely on snails for

18848 hours with no additional food source. At the end of the 48-hour assay period fish were

189 removed from trial tanks, photographed, and placed back into mixed-sex stock tanks. We then

190 recorded the number of snails that were consumed (empty shells remaining) and unconsumed.

191 Finally, we measured the size of each snail shell from the anterior tip of the shell's aperture to

192 farthest tip of the spire (mm) using digital calipers and image processing software . In total, we 193 measured feeding success for 13 outgroup generalists, 20 generalists, 55 durophages, 20 scale- 
194 eaters, 25 F1 hybrids, and 63 F2 hybrids. Out of the 196 trials, only 11 finished the trial period

195 with four snail shells instead of the given five, suggesting that at most $3.5 \%$ of snail consumption

196 involved also eating the shell.

\section{Data Processing}

199 No differences between fully consumed and partially consumed snails

200 We noticed that a portion of the snails were only partially consumed (i.e. part of the snail tissue

201 remained in the shell versus a completely empty shell after 48 hours) and therefore used a

202 generalized linear mixed model (GLMM) with a binomial response distribution to determine if

203 partially consumed snails should be analyzed separately from fully consumed snails. We

204 included 1) whether snails were fully or partially consumed as the response variable (binomial

205 data), 2) species designation as a fixed effect, 3) population and fish ID as random effects, and 4)

$206 \log$ standard length as a covariate. We found that the pattern of partially and fully consumed

207 snails did not vary across species $(\chi 2=2.73, d f=5, P=0.74)$, and therefore included all partially

208 consumed snails in the general "consumed" category for the remainder of our analyses.

210 Statistical Analysis

211 We used a linear mixed model to investigate the relationship between nasal protrusion distance

212 and species. For this analysis we used a subset of our data which includes: 9 generalists, 50

213 durophages, 17 F1 hybrids, and 62 F2 hybrids. Our model included 1) log nasal protrusion size

214 as the response variable, 2) species designation, log standard length, and their interaction as fixed

215 effects, and 3) population as a random effect. We also used Tukey’s HSD to make post hoc

216 comparisons across species. 
We used a GLMM with a negative binomial distribution to explore whether the number

218 of snails consumed varied between species. We included 1) whether snails were consumed or

219 unconsumed as the response variable (binomial data), 2) species designation as a fixed effect, 3)

220 population and fish ID as random effects, and 4) log standard length as a covariate. We made

221 additional post hoc comparisons between groups using Tukey’s HSD.

222 We used a linear mixed model to determine if the size of snails varied by whether they

223 were consumed or unconsumed and whether that varied between species. We included 1) snail

224 size $(\mathrm{mm})$ as the response variable, 2) whether snails were consumed or unconsumed, species

225 designation, and their interaction as fixed effects, 3) population and fish ID as random effects,

226 and log standard length as a covariate. We made additional post hoc comparisons between

227 groups using contrasts and an FDR correction.

Finally, we investigated if nasal protrusion distance affected the maximum size snail an

229 individual could consume as an estimate of snail-shelling performance. For this analysis we only

230 considered purebred durophages and F2 hybrids (separately) as they had the largest observed

231 variance in nasal protrusion size and only included individuals that consumed at least one snail

232 during the feeding trial. For each group, we used a linear model with 1) the size of the largest

233 consumed snail for each individual as the response variable, 2) log nasal protrusion size, log

234 standard size, and their interaction as fixed effects, and 3) the residuals from a linear model

235 investigating the relationship between snail size and nasal protrusion size as a covariate. We

236 included this additional covariate because we found a strong positive relationship between mean

237 snail size provided during trials and nasal protrusion in both purebred durophages (LM: $P=1.72 \mathrm{x}$

$23810^{-9}$, adjusted $\left.\mathrm{R}^{2}=0.14\right)$ and $\mathrm{F} 2$ hybrids $\left(\mathrm{LM}: P=5.58 \times 10^{-10}\right.$, adjusted $\left.\mathrm{R}^{2}=0.12\right)$, and wanted to

239 account for this variation in the model (Figure S2). This variation reflected our attempt to 
240 provide some larger snails in trials with larger fish to better assess performance. We additionally

241 included the random effect of population in our durophage model.

243 Ethical Statement

244 This study was conducted with the approval of the Animal Care and Use Committee of the

245 University of North Carolina, Chapel Hill, NC (protocol\# 15-179.0). All wild fish were collected

246 with a research and export permit from the Bahamas BEST commission, renewed annually since 2472011.

249 Results

250 Nasal protrusion size does not vary between purebred durophages and hybrids

251 Our linear mixed model indicated that nasal protrusion size is significantly associated with log

252 standard length $\left(\chi 2=27.63, d f=1, P=1.47 \times 10^{-7}\right)$, but that this relationship does not vary between

253 purebred and hybrid durophages $(\chi 2=3.22, d f=3, P=0.36$; Figure 1 A \& S1). Post hoc analysis

254 indicated that generalists had smaller noses than durophages $(P<0.0001)$ and $\mathrm{F} 1$ hybrids $(P=$ $2550.016)$. 
A.

C.

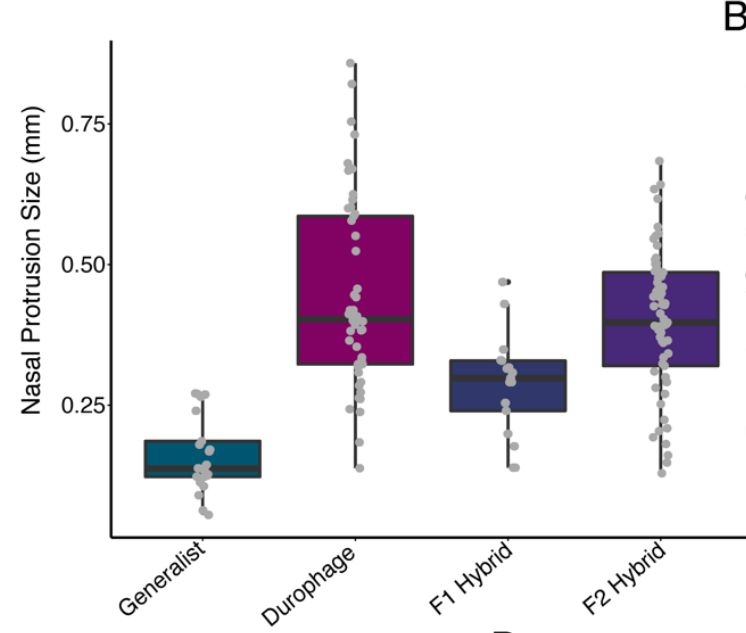

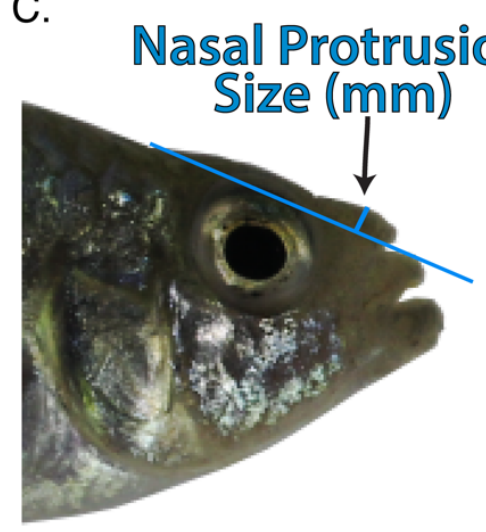

B.

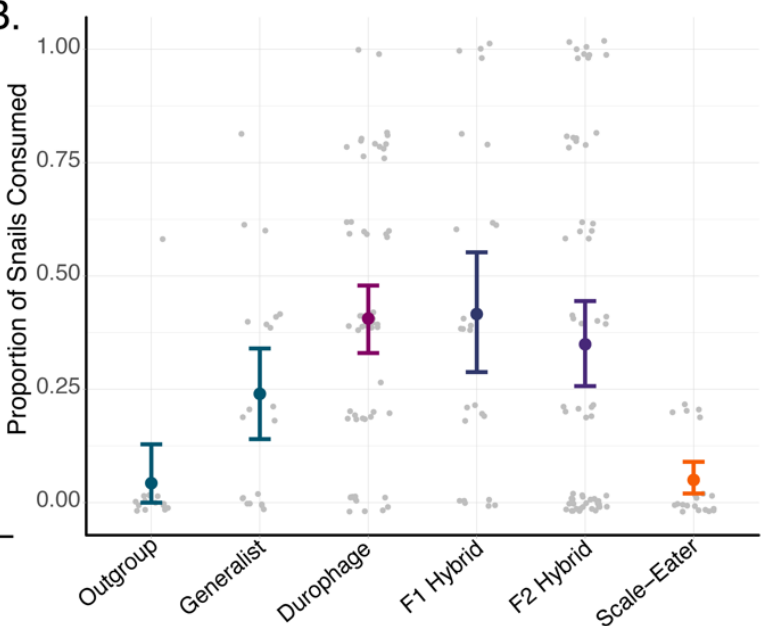

D. Outgroup

\section{(100)}

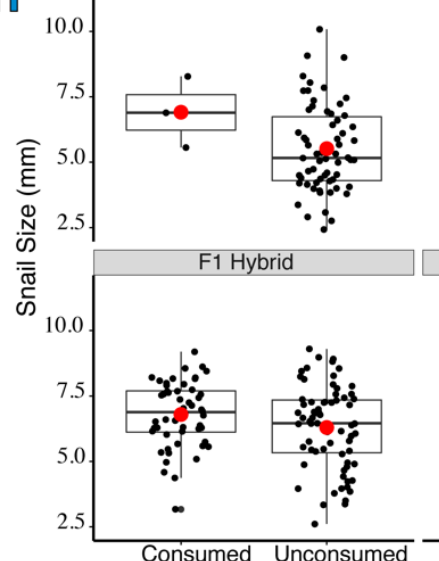

Generalist
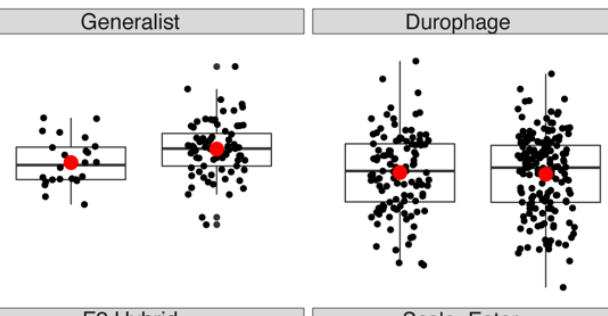

F2 Hybrid Scale-Eater
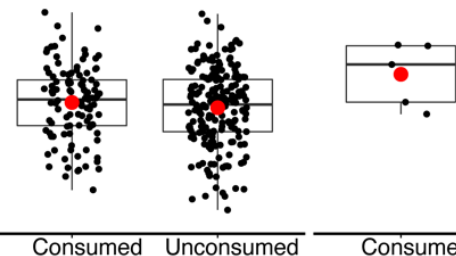
$\because$.

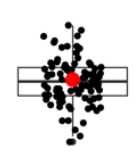

Figure 1. Snail consumption, nasal protrusion size, and snail size by species. A) Variation in

nasal protrusion size across pupfish groups. Grey dots represent individual fish. B) Proportion of snails consumed across six groups of pupfish. Colored dots represent mean proportion, and error bars represent 95\% confidence intervals (bootstrapping: 1,000 iterations). C) Visualization of how nasal protrusion size was measured (pictured: durophagous pupfish). D) Visualization of the size of consumed and unconsumed snails for each species. Black dots represent individual snails and red dots represent the mean snail size. 
Purebred durophages and their hybrids consume the most snails

268 We found that species designation was a significant predictor for the number of snails an

269 individual consumed (GLMM; $\left.\chi 2=35.61, d f=5, P=1.129 \times 10^{-6}\right)$. Specifically, we found that

270 durophages, F1 hybrids, and F2 hybrids consumed more snails than the generalist outgroup

271 population (Lake Cunningham, New Providence Island, Bahamas) and scale-eating pupfish

272 (Figure 1B). Durophages, F1 hybrids, and F2 hybrids also consumed twice as many snails as

273 generalists, however this difference was not significant.

Consumed snails were larger than unconsumed snails

276 In general, we found that the size of snails varied 1$)$ by whether they were consumed $(\chi 2=4.002$,

$277 d f=1, P=0.045)$, and 2$)$ across species $(\chi 2=24.79, d f=5, P=0.00015$; Figure S1). Specifically, we

278 found that consumed snails were on average $0.12 \mathrm{~mm}$ larger in diameter than unconsumed snails

$279(P=0.046)$. Generalists and scale-eaters received snails that were approximately $17 \%$ larger than

280 other groups (generalists: $P=0.016$; scale-eaters: $P=0.02$ ). Although this was unintentional due to

281 the available size distributions of snails in our colony over the ten month course of the feeding

282 trails, we believe that it did not introduce a significant bias because 1) larger snails were more

283 likely to be consumed (in fact there was only an $8 \%$ difference between the mean size of snail

284 given to generalists and scale-eaters $v s$ the mean size of consumed snails) and 2) generalists and

285 scale-eaters were excluded from analyses which examined how nasal protrusion affected a fish's

286 ability to consume snails.

287

290 We found no effect of log nasal protrusion size, log standard length, or their interaction on the

291 size of the largest consumed snail for either durophages $\left(P_{\log (\text { nasalprotrusionsize })}=0.49\right.$, 
bioRxiv preprint doi: https://doi.org/10.1101/2020.03.23.004416; this version posted March 26, 2020. The copyright holder for this preprint (which was not certified by peer review) is the author/funder. All rights reserved. No reuse allowed without permission.

$\left.292 P_{\log (\text { standardlength })}=0.61, P_{\text {interaction }}=0.56\right)$ or F2 hybrids $\left(P_{\log (\text { nasalprotrusionsize })}=0.83\right.$,

$293 P_{\log (\text { standardlength })}=0.66, P_{\text {interaction }}=0.91 ;$ Figure 2$)$.

294 


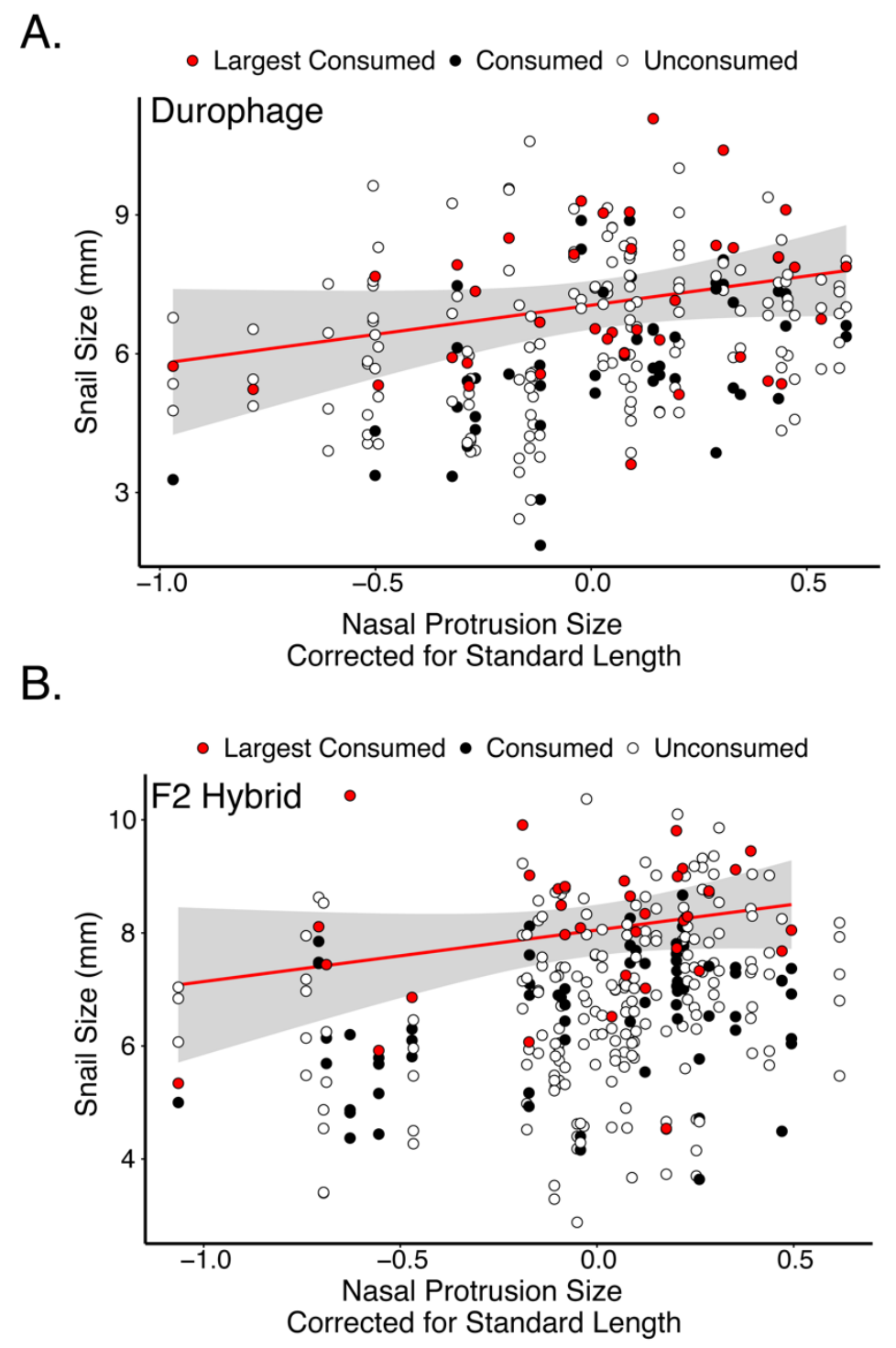

297 Figure 2. The maximum prey size a pupfish can consume was not affected by nasal

298 protrusion size. The $\mathrm{X}$-axis shows nasal protrusion size corrected for standard length while the

299 Y-axis shows snail size $(\mathrm{mm})$. Red dots show the size of largest consumed snail from each trial,

300 the red line represents the linear model describing the relationship between nasal protrusion size

301 and the largest consumed snails, and the grey area represents 95\% CI. Closed circles show the

302 size of other snails that were consumed during trials; open circles show the size of unconsumed 303 snails. 


\section{Discussion}

305 We present the first strong evidence in any cyprinodontiform fish that the durophagous pupfish is 306 an oral sheller, shaking snails free from their shells rather than crushing or ingesting the whole

307 shell. This is consistent with their notably non-molariform pharyngeal jaws relative to generalists

308 and snail-crushing species (Figure 3). We then tested the hypothesis that the durophagous

309 pupfish's novel nasal protrusion is an adaptation for removing snails from their shells, potentially

310 functioning as a fulcrum. We predicted that durophagous pupfish would 1) consume more snails

311 than other groups, and 2) consume larger snails than other groups. We found that both

312 durophages and their F1 and F2 hybrid offspring consumed the most snails compared to other

313 groups (Figure 1B), indicating that any substantial amount of durophagous genetic ancestry

314 increases the number of snails consumed over a 48-hour feeding trial. However, contrary to our

315 expectations, we found no significant evidence that larger nasal protrusions within hybrid or

316 parental durophagous pupfish populations enabled the fish to consume larger snails (Figure 2).

Durophages have a stronger behavioral preference for snails compared to other species

319 One explanation for the observed pattern is that durophagous pupfish have a stronger preference

320 for snails which is independent from their novel nasal protrusion. We see some support for this

321 within our data. Generalist pupfish from San Salvador Island consumed significantly more snails

322 than generalists found outside of the radiation on New Providence Island, and even consumed

323 statistically similar amounts of snails as purebred durophages despite having much smaller nasal

324 protrusions (Figure 1A\&B). It could be that extensive geneflow between generalists and

325 durophages on San Salvador Island spread alleles for snail-eating preference throughout both

326 pupfish species (Martin and Feinstein 2014). Alternatively, the common ancestor of durophages 
327 and generalists may have had a strong preference for snails (Martin and Feinstein 2014; Richards

328 and Martin 2017). The increased aggression of both male and female durophages toward

329 conspecifics by potentially alternate genetic pathways to scale-eaters, as shown in a recent study

330 (St. John et al. 2019), could also be associated with their stronger preference for aggressively

331 attacking snails to flip them over before gripping the body of the snail in their oral jaws and

332 shaking them free from their shells (Supplemental Video 1).

333 Liem's hypothesis and subsequent work has long supported the idea that morphological

334 specialization need not coincide with trophic specialization, or vice versa. For example,

335 Tropheops tropheops and Metriaclima zebra, two cichlids from Lake Malawi that are

336 morphologically specialized for scraping algae often fill a generalist ecological niche, consuming

337 zooplankton, benthic invertebrates, and phytoplankton (Liem 1978, 1980; McKaye and Marsh

338 1983), particularly during periods of resource abundance (Martin and Genner 2009). An

339 analogous argument can be made for individual dietary specialization within a population

340 (Bolnick et al. 2003). For example, Werner and Sherry (1987) found that individual Cocos

341 Island finches specialize on a wide variety of taxa including crustacea, nectar, fruit, seeds,

342 mollusks, and lizards, and that individual dietary specialization was most likely driven by

343 behavioral differences. Similarly, increased levels of individual specialization in sticklebacks are

344 driven by shifts in forager density or intraspecific competition (Svanbäck and Bolnick 2005,

345 2007; Araújo et al. 2008). Thus, individual specialization is often driven entirely by differences

346 in behavior, feeding preference, or other external factors and can be divorced from adaptive

347 differences in morphology (Werner and Sherry 1987).

349 Alternative functions of the novel nasal protrusion 
350 We investigated whether an increase in nasal protrusion size affected the maximum size snail an

351 individual could consume (Figure 2). However, it could be that the novel nasal protrusion is

352 related to feeding efficiency, e.g. in handling time per snail, or is a sensory organ used for

353 locating snails more efficiently with potentially increased numbers of superficial neuromasts

354 (Shibuya et al. 2019). There are several examples of nasal protrusions that are used for this

355 purpose. The unique rostrums of paddlefish (Polydontidae), sturgeon (Acipenseridae), and

356 sawfish (Pristidae) are all used as sensory organs, containing electroreceptors, lateral line canals,

357 and even barbels for detecting prey items (Miller 2006; Wueringer et al. 2012). The novel nasal

358 protrusion of the durophagous pupfish may also be a sensory organ, however, whether the nasal

359 protrusion has an increased number of superficial neuromasts is still unknown.

360 Alternatively, the novel nasal protrusion may allow durophagous pupfish to orally shell

361 snails more quickly, increasing their feeding efficiency. For example, Schluter (1993)

362 documented that benthic sticklebacks with deep bodies, large mouths, and few, short gill rakers

363 were more efficient at consuming benthic prey items, while limnetic species of stickleback, with

364 slender bodies, small mouths, and many, long gill rakers, were more efficient at consuming

365 limnetic prey items. Interestingly, Schluter (1993, 1995) also found that F1 hybrids had

366 decreased efficiency feeding on both limnetic and benthic prey items which was primarily due to

367 their intermediate phenotypes and suggested that reduced fitness in hybrids helps maintain

368 species boundaries between benthic and limnetic species. It could be that the durophage F1 and

369 F2 hybrids have similar preferences for gastropods, but cannot consume snails as efficiently due

370 to their intermediate phenotype. However, we found no strong evidence suggesting that the nasal

371 protrusion is adapted for oral shelling (Figure 2). Future work should investigate other traits that

372 may be adaptive for oral shelling such as the strength of the dorsal head of the maxilla which 
373 comprises the skeletal basis of the novel nasal protrusion, structural differences in the

374 mandibular symphysis, coronoid process, or the articular bones which may all provide additional

375 strength or stabilization during biting, or tooth variation in the durophage pharyngeal jaws (Fig.

376 3). Indeed, there is subtle variation apparent in the pharyngeal teeth and jaws of durophages

377 compared to other pupfish species (Figure 3) which has not been previously reported, suggesting

378 that pharyngeal jaws may be adapted for processing hard-shelled prey. 


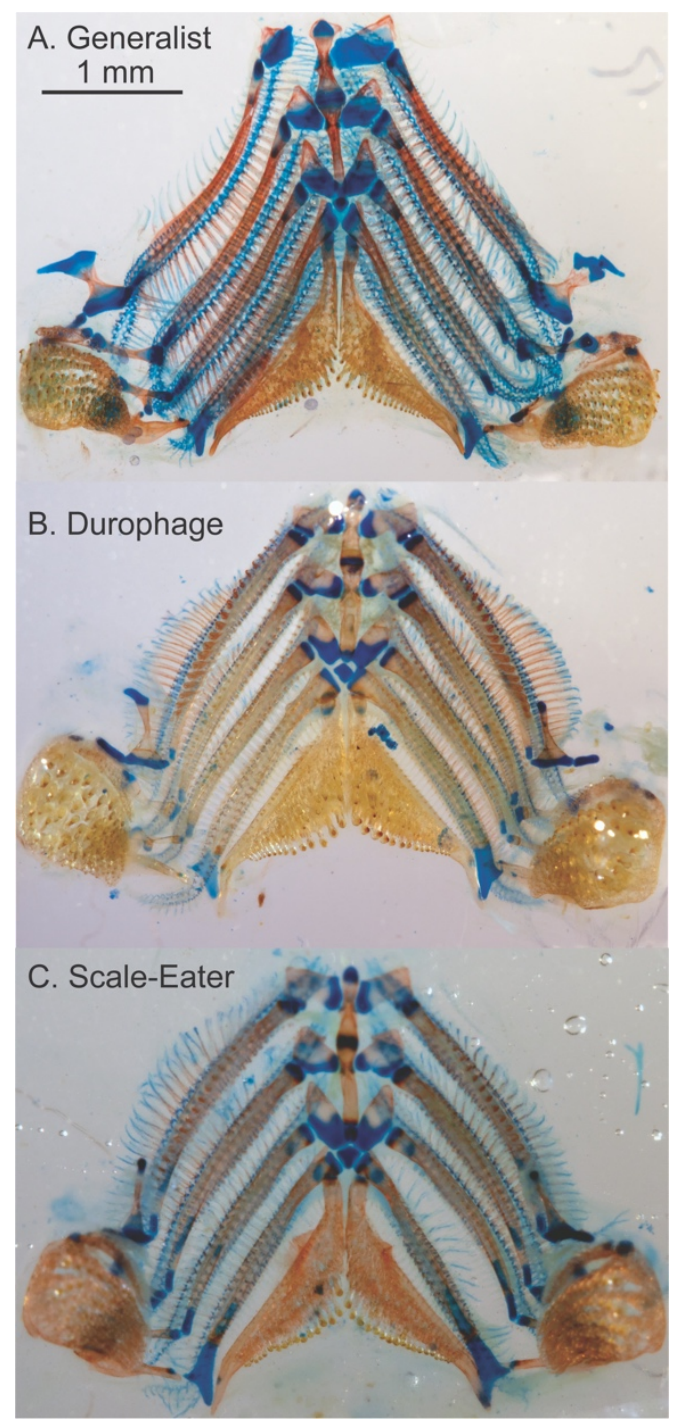

383 Image of the dissected branchial skeleton and pharyngeal jaws of A) generalist, B) durophage, 384 and C) scale-eater pupfish. Scale $(1 \mathrm{~mm})$ is shown in Figure A and is consistent across all three 385 photos. From these three individuals, the representative snail-eater has lower pharyngeal teeth 386 that are $50 \%$ longer and $75 \%$ wider than the generalist or scale-eating individuals. 
The novel nasal protrusion may be a sexually selected trait

391 Finally, the novel nasal protrusion may be unrelated to oral shelling and instead may be used in

392 species recognition or mate preference functions. Exaggerated traits, like the novel nasal

393 protrusion in durophage pupfish, commonly arise via sexual selection. For example, forceps size

394 in earwigs (Simmons and Tomkins 1996), major claw size in fiddler crabs (Rosenberg 2002),

395 and the size of the sword tail ornament present in swordtail fish (Rosenthal and Evans 1998) are

396 all thought to be sexually selected traits. Two commonly invoked hallmarks of a sexually

397 selected trait are 1) allometric scaling compared to body size and 2) that the trait is sexually

398 dimorphic (Kodric-Brown and Brown 1984; Kodric-Brown et al. 2006; Shingleton and Frankino

399 2013). In pupfish, there is a weak positive relationship between standard length and nasal

400 protrusion size observed for generalists (Figure S1A, generalist slope $=0.35)$. Generalist pupfish

401 mostly likely resemble the most recent common ancestor for the radiation, making the observed

402 slope a good null expectation for how nasal protrusion size should scale with body size in

403 pupfish. In durophages, we observe much stronger positive allometry of the nasal protrusion

404 (Figure $\mathrm{S} 1 \mathrm{~B}$, durophage slope $=0.93$ ), in which large durophage individuals have nasal protrusion

405 sizes more than twice as large as those in large generalists. However, we found no significant

406 difference in nasal protrusion size between male and female durophages when accounting for

407 these size differences (LM, $P=0.96)$.

\section{Conclusion}

410 In conclusion, we did not find evidence to support that the novel nasal protrusion observed in

411 durophagous pupfish is adapted for consuming large snails. Instead, we found that purebred

412 durophages and their F1 and F2 hybrids have stronger preferences for consuming snails than 
413 other species. We suggest that the novel nasal protrusion may be adapted for other aspects of oral

414 shelling such as feeding efficiency, or that variation in other traits, such as the pharyngeal jaws

415 (Figure 3), may play a larger role in oral shelling. Alternatively, this may be an example of

416 trophic specialization due to behavioral specialization (i.e. feeding preference).

417 


\section{References}

419 Araújo, M. S., P. R. Guimarães, R. Svanbäck, A. Pinheiro, P. Guuimarães, S. F. Dos Reis, and D.

420 I. Bolnick. 2008. Network analysis reveals contrasting effects of intraspecific competition on

421 individual vs. population diets. Ecology 89:1981-1993.

422 Bolnick, D. I., R. Svanbäck, J. A. Fordyce, L. H. Yang, J. M. Davis, C. D. Hulsey, and M. L.

423 Forister. 2003. The ecology of individuals: incidence and implications of individual

424 specialization. The American naturalist 161:1-28.

425 Conith, M. R., A. J. Conith, and R. C. Albertson. 2019. Evolution of a soft-tissue foraging 426 adaptation in African cichlids: Roles for novelty, convergence, and constraint. Evolution 20724272084.

428 Conith, M. R., Y. Hu, A. J. Conith, M. A. Maginnis, J. F. Webb, and R. Craig Albertson. 2018.

429 Genetic and developmental origins of a unique foraging adaptation in a Lake Malawi cichlid 430 genus. Proceedings of the National Academy of Sciences of the United States of America $431 \quad 115: 7063-7068$.

432 De Visser, J., and C. D. N. Barel. 1996. Architectonic constraints on the hyoid's optimal starting 433 position for suction feeding of fish. Journal of Morphology 228:1-18.

434 Futuyman, D. J., and G. Moreno. 1988. The Evolution of Ecological Specialization. Annual

435 Review of Ecology and Systematics 19:207-233.

436 Gidmark, N. J., N. Konow, E. LoPresti, and E. L. Brainerd. 2013. Bite force is limited by the

437 force-length relationship of skeletal muscle in black carp, Mylopharyngodon piceus. Biology 438 Letters 9.

439 Gidmark, N. J., C. Taylor, E. Lopresti, and E. Brainerd. 2015. Functional morphology of 440 durophagy in black carp, Mylopharyngodon piceus. Journal of Morphology 276:1422-1432. 
441 Grubich, J. 2003. Morphological convergence of pharyngeal jaw structure in durophagous

442 perciform fish. Biological Journal of the Linnean Society 80:147-165.

443 Hagey, F. M., and J. E. Mylroie. 1995. Pleistocene lake and lagoon deposits, San Salvador

444 island, Bahamas. Special Papers-Geological Society of America 77-90.

445 Hernandez, L. P., D. Adriaens, C. H. Martin, P. C. Wainwright, B. Masschaele, and M. Dierick.

446 2018. Building trophic specializations that result in substantial niche partitioning within a young

447 adaptive radiation. Journal of Anatomy 232:173-185.

448 Kodric-Brown, A., and J. H. Brown. 1984. Truth in advertising: the kinds of traits favored by

449 sexual selection. American Naturalist 124:309-323.

450 Kodric-Brown, A., R. M. Sibly, and J. H. Brown. 2006. The allometry of ornaments and

451 weapons. Proceedings of the National Academy of Sciences of the United States of America

$452 \quad 103: 8733-8738$.

453 Konings, A. 2007. Malawi cichlids in their natural habitat 4th Edition.

454 Lauder, G. V. 1983. Functional and morphological bases of trophic specialization in sunfishes

455 (Teleostei, centrarchidae). Journal of Morphology 178:1-21.

456 Lencer, E. S., W. C. Warren, R. Harrison, and A. R. McCune. 2017. The Cyprinodon variegatus

457 genome reveals gene expression changes underlying differences in skull morphology among

458 closely related species. BMC Genomics 18:424.

459 Liem, K. F. 1978. Modulatory multiplicity in the functional repertoire of the feeding mechanism

460 in cichlid fishes. I. Piscivores. Journal of Morphology 158:323-360.

461 Liem, K. F. 1980. Adaptive significance of intra- and interspecific differences in the feeding

462 repertoires of cichlid fishes. Integrative and Comparative Biology 20:295-314.

463 Lundeba, M., J. S. Likongwe, H. Madsen, and J. R. Stauffer. 2011. Oral shelling of Bulinus spp. 
464 (Mollusca: Planorbidae) by the Lake Malaŵi cichlid, Metriaclima lanisticola (Pisces: Cichlidae).

465 Journal of Freshwater Ecology 26:593-597.

466 Madsen, H., K. C. J. Kamanga, J. R. Stauffer, and J. Likongwe. 2010. Biology of the

467 molluscivorous fish trematocranus placodon (pisces: Cichlidae) from lake malaŵi. Journal of

468 Freshwater Ecology 25:449-455.

469 Martin, C. H. 2016. The cryptic origins of evolutionary novelty: 1000-fold faster trophic

470 diversification rates without increased ecological opportunity or hybrid swarm. Evolution

$471 \quad 70: 2504-2519$.

472 Martin, C. H., and L. C. Feinstein. 2014. Novel trophic niches drive variable progress towards

473 ecological speciation within an adaptive radiation of pupfishes. Molecular Ecology 23:1846-

4741862.

475 Martin, C. H., and M. J. Genner. 2009. High niche overlap between two successfully coexisting

476 pairs of Lake Malawi cichlid fishes. Canadian Journal of Fisheries and Aquatic Sciences 66:579477588.

478 Martin, C. H., and P. C. Wainwright. 2011. Trophic novelty is linked to exceptional rates of

479 morphological diversification in two adaptive radiations of cyprinodon pupfish. Evolution

$480 \quad 65: 2197-2212$

481 2013a. A Remarkable Species Flock of Cyprinodon Pupfishes Endemic to San Salvador

482 Island, Bahamas. Bulletin of the Peabody Museum of Natural History 54:231-241.

483 2013b. On the Measurement of Ecological Novelty: Scale-Eating Pupfish Are Separated

484 by 168 my from Other Scale-Eating Fishes. PLoS ONE 8:e71164.

485 McKaye, K. R., and A. Marsh. 1983. Food switching by two specialized algae-scraping cichlid

486 fishes in Lake Malawi, Africa. Oecologia 56:245-248. 
487 Miller, M. J. 2006. The Ecology and Functional Morphology of Feeding of North American

488 Sturgeon and Paddlefish. Pages 87-102 inSturgeons and Paddlefish of North America.

489 Pyke, G. H. 1984. Optimal foraging theory: a critical review. Annual review of ecology and

490 systematics. Vol. 15 15:523-575.

491 Ralston, K. R., and P. C. Wainwright. 1997. Functional consequences of trophic specialization in

492 pufferfishes. Functional Ecology 11:43-52.

493 Richards, E., and C. Martin. 2017. Adaptive introgression from distant Caribbean islands

494 contributed to the diversification of a microendemic radiation of trophic specialist pupfishes.

495 PLOS Genetics.

496 Robinson, B. W., and D. S. Wilson. 1998. Optimal foraging, specialization, and a solution to

497 Liem's paradox. American Naturalist 151:223-235.

498 Rosenberg, M. S. 2002. Fiddler crab claw shape variation: A geometric morphometric analysis

499 across the genus Uca (Crustacea: Brachyura: Ocypodidae). Biological Journal of the Linnean

$500 \quad$ Society $75: 147-162$.

501 Rosenthal, G. G., and C. S. Evans. 1998. Female preference for swords in Xiphophorus helleri

502 reflects a bias for large apparent size. Proceedings of the National Academy of Sciences of the

503 United States of America 95:4431-4436.

504 Schindelin, J., I. Arganda-Carreras, E. Frise, V. Kaynig, M. Longair, T. Pietzsch, S. Preibisch, et

505 al. 2012. Fiji: an open-source platform for biological-image analysis. Nature Methods 9:676-

506682.

507 Schluter, D. 1993. Adaptive radiation in sticklebacks: size, shape, and habitat use efficiency.

508 Ecology 74:699-709.

509 1995. Adaptive Radiation in Sticklebacks: Trade-Offs in Feeding Performance and 
510 Growth. Ecology 76:82-90.

511 Shibuya, A., J. Zuanon, and M. R. de Carvalho. 2019. Neuromast distribution and its relevance

512 to feeding in Neotropical freshwater stingrays (Elasmobranchii: Potamotrygonidae).

513 Zoomorphology.

514 Shingleton, A. W., and W. A. Frankino. 2013. New perspectives on the evolution of exaggerated

515 traits. BioEssays 35:100-107.

516 Simmons, L. W., and J. L. Tomkins. 1996. Sexual selection and the allometry of earwig forceps.

517 Evolutionary Ecology 10:97-104.

518 Slootweg, R. 1987. Prey selection by molluscivorous cichlids foraging on a schistosomiasis

519 vector snail, Biomphalaria glabrata. Oecologia 74:193-202.

520 St. John, M. E., J. A. McGirr, and C. H. Martin. 2019. The behavioral origins of novelty: did

521 increased aggression lead to scale-eating in pupfishes? Behavioral Ecology 30:557-569.

522 Svanbäck, R., and D. I. Bolnick. 2005. Intraspecific competition affects the strength of individual

523 specialization: An optimal diet theory method. Evolutionary Ecology Research 7:993-1012.

$524-$ 2007. Intraspecific competition drives increased resource use diversity within a natural

525 population. Proceedings of the Royal Society B: Biological Sciences 274:839-844.

526 Vranken, N., M. Van Steenberge, and J. Snoeks. 2019. Similar ecology, different morphology:

527 Three new species of oral-mollusc shellers from Lake Edward. Journal of Fish Biology.

528 Wainwright, P. C. 1987. Biomechanical limits to ecological performance: mollusc-crushing by

529 the Caribbean hogfish, Lachnolaimus maximus (Labridae). Journal of Zoology 213:283-297.

530 Wainwright, P. C. 1991. Ecomorphology: Experimental functional anatomy for ecological

531 problems. Integrative and Comparative Biology 31:680-693.

532 Werner, T. K., and T. W. Sherry. 1987. Behavioral feeding specialization in Pinaroloxias 
533 inornata, the "Darwin's Finch" of Cocos Island, Costa Rica. Proceedings of the National

534 Academy of Sciences 84:5506-5510.

535 Winterbottom, R. 1974. The familial phylogeny of the Tetraodontiformes (Acanthopterygii:

536 Pisces) as evidenced by their comparative myology. Smithsonian Contributions to Zoology.

537 Wueringer, B. E., L. Squire, S. M. Kajiura, N. S. Hart, and S. P. Collin. 2012. The function of

538 the sawfish's saw. Current Biology.

539 